\title{
Restoration for Motion Blurred Images of Moving Objects
}

\author{
Guixiang Zhou \\ Department of mathematics, Xinxiang University, Xinxiang 453003, China \\ zhouguixiang2010@yahoo.cn
}

Keywords: Restoration, motion blurred image, point spread function

\begin{abstract}
Image restoration plays an important role in transportation applications. This paper studies a motion blurred image processing method, which has good recovery effect. In this method, first the wiener filter is used for image restoration. Then, based on the error parameter analysis, the parameters of point spread function are estimated, and the noise parameter is estimated by using the probability and statistics method. Furthermore, the ringing effect is processed by using the smooth boundary method. Finally, experimental results show that the proposed method can restore the motion blurred images effectively and has strong robustness for the noise.
\end{abstract}

\section{Introduction}

In the actual environment, the images of vehicles obtained by the camera, due to the relative movement between the vehicle and the camera, often become blur. In order to effectively use these images, It is necessary to restore the motion blurred image so as to get better visual effect of image. The common method of the restoration of motion blurred image is Wiener filtering [1,2], parameter estimation is the key of the blurred image restoration, and the Literature [3] use artificial methods to select the noise-signal power ratio parameter in Wiener filtering. The Literature [4] use the method of the image spatial gradient to estimate the length parameter of point spread function. The Literature [5] on the spectrum of the motion blurred image is accumulated enhancement, and then it estimates the length parameter of the point spread function by observation.

This paper introduces a method for motion blurred images restoration. Though using the Wiener filtering to restoration and the error parameter analysis, this paper estimate the length parameter of point spread function. Based on the probability statistics method, the paper obtained the estimation of noise parameters and the processing of the ringing effects. the experimental results show that, the method at low SNR can still accurately estimate the blurring length parameter, is robust to the noise-signal power ratio, on motion blurred image with good restoration effect.

Image restoration is important in traffic information acquisition. This paper introduces a method for motion blurred License plate images restoration. Wiener filter is used to restore the motion blurred images. The parameters of point spread function are estimated by using error parameter analysis, while the noise parameter is estimated based on probability and statistics method. Furthermore, the ringing effect is processed. Experimental results show that the proposed method can restore the motion blurred images effectively and is robust to the noise.

The remainder of this paper is organized as follows. Section II presents the image degradation and restoration model. Section III proposes how to estimate the point spread function parameter and the noise level parameter. Section IV presents how to process of the ringing effects. Experimental results are given in Section V, while Section VI concludes this paper.

\section{Image Degradation and Restoration Model}

The model of the Motion blurred images degradation can be described

$$
g(x, y)=h(x, y) * f(x, y)+n(x, y)
$$

where $f(x, y)$ is the original image, $g(x, y)$ is the blurred images, $h(x, y)$ is the point spread function, and $n(x, y)$ is the additive noise. 


$$
G(u, v)=H(u, v) F(u, v)+N(u, v)
$$

where equation (2) is the expression of (1) in the frequency domain. The Capital letters is the Fourier transformation of the correspondingly original function.

Using the Wiener filtering, the frequency domain model for image restoration is:

$$
F(u, v)=\left[\frac{1}{H(u, v)} \frac{|H(u, v)|^{2}}{|H(u, v)|^{2}+\frac{S_{n}(u, v)}{S_{f}(u, v)}}\right] G(u, v)
$$

where $|H(u, v)|^{2}=\mathbf{H}^{*}(u, v) H(u, v)$, and $\mathbf{H}^{*}(u, v)$ is the Complex conjugate of $H(u, v) . S_{n}(u, v)$ is the noise power spectrum and $S_{f}(u, v)$ is non-degraded image power spectrum.

\section{The Parameter Estimation}

Point Spread Function Estimation. The point spread function of motion blur can be expressed as:

$$
h(x, y)= \begin{cases}\frac{1}{d}, & 0 \leq x \leq d, y=0 \\ 0, & \text { others }\end{cases}
$$

where equation (4) is a point spread function of motion blur in the horizontal direction, $d$ is a motion blur length. If the motion blur is not in the horizontal direction, then it can conversion to the horizontal direction via coordinate.

In this paper, it used the error-parameter analysis [6] to identify the point spread function. This method in the observation of the images which signal to noise ratio is low and motion blur length is larger can also get a better estimate effect. The method of Error-parameter analysis uses the error metric $E$ to estimate the parameter $d$, and the steps are as follows:

(1) Selecting a length parameter to search the range and be represent by the initial length value $d_{0}$ , the length of step $\Delta d$ and the number of searching step $\mathrm{k}$ to represent.

(2) Obtain the point spread function $h$ by the parameter $d$, implemented the restoration algorithm, and determine the estimation $f$ of the restoration images based on $h$ and the observed image $g$. Compute the reconstruction error $e=\left\|g-f^{\prime} \times h\right\|^{2}$ or the frequency domain error $E=\left\|G-F^{\prime} H\right\|^{2}$.

(3) Make a $E-d$ curve, in which the rate of change will be significantly smaller near the real value of $d$, and then estimate the parameter $d$ of the point spread function and obtain the corresponding point spread function.

In order to get a more accurate estimation of value, this paper usually set several different signal to noise ratio parameters in order to obtain a number of curves and facilitate the curve analysis and observation. In the estimation of the parameter $d$ of the point spread function, select the flat position in the $E-d$ curve as the estimated value. In the estimation of the range of the point spread function, it generally cannot be very precise. When setting the searching range is very large, sometimes there will be several possible estimation values. So it's necessary to select it through experiments.

The Noise Parameter Estimation. Because of the affection by many factors on the actual images, it is difficult to obtain the parameters of noise-signal power ratio in the models of Wiener filtering restoration. We can use the degraded image estimation noise-signal power ratio [7] to calculate the local variance of degraded image, select the maximum of the local variance as image variance $2 f$, find a flat area in the image, in which make the average of the local variance as the estimation of the noise variance $2 \mathrm{n}$, and then make the ration of noise variance and image variance as the estimation of 
the noise-signal power ratio $S_{n}(u, v) / S_{f}(u, v)$. If there is no suitable flat area, it can use the ration of the minimum value and the maximum value of images local variance as the estimation of the image noise-signal power ratio $S_{n}(u, v) / S_{f}(u, v)$.

In practical application, because of the flat area for rationality, the type of the noise and so on, it is need to amend the parameters of the image noise-signal power ratio.

\section{The process of the ringing effect}

When the Image is restored, there is the interference stripes in the places which image gray transform violently, we called it ringing effect. If it cannot be processed, there is a great effect on the restored results. In the discrete Flushier transformation, there is need to sought image data cyclical, but the actual image can not meet this need. It must be added pixels in image edge to meet the need for Flushier transformation. If it uses Wiener filtering directly, the extended portion of pixels is considered as 0 by default, which will bring the great edge error. Therefore, it can solve this problem through the use of smoothing method.

$$
\begin{aligned}
& \text { Set the original image is }\left[\begin{array}{ccc}
a & \cdots & b \\
\vdots & & \vdots \\
c & \cdots & d
\end{array}\right] \text {, and the image after smoothing edges is } \\
& \qquad\left[\begin{array}{cccccc}
a & \cdots & b & b & \cdots & b \\
\vdots & & \vdots & \vdots & & \vdots \\
c & \cdots & d & d & \cdots & d \\
c & \cdots & d & d & \cdots & d \\
\vdots & & \vdots & \vdots & & \vdots \\
c & \cdots & d & d & \cdots & d
\end{array}\right]
\end{aligned}
$$

According to this rule will image expansion, so that both can meet the requirements of the Fourier transform, but also can reduce the gray-scale transformation of sharp edge, suppressed ringing effect.

\section{Experimental Results}

To validate the proposed method, we test our proposed method on real motion blurred images. Figure 1 and 2 show the restored results using our proposed method. Form the results, we can see that our proposed method can get good visual quality.

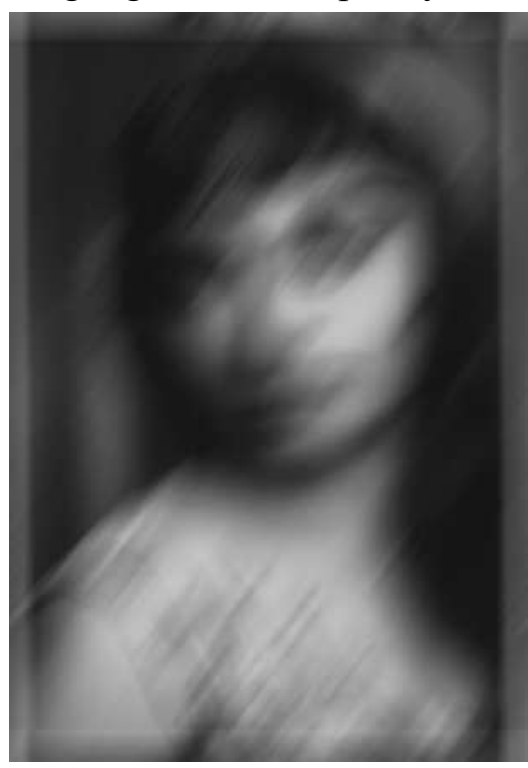

(a) Blurred Image

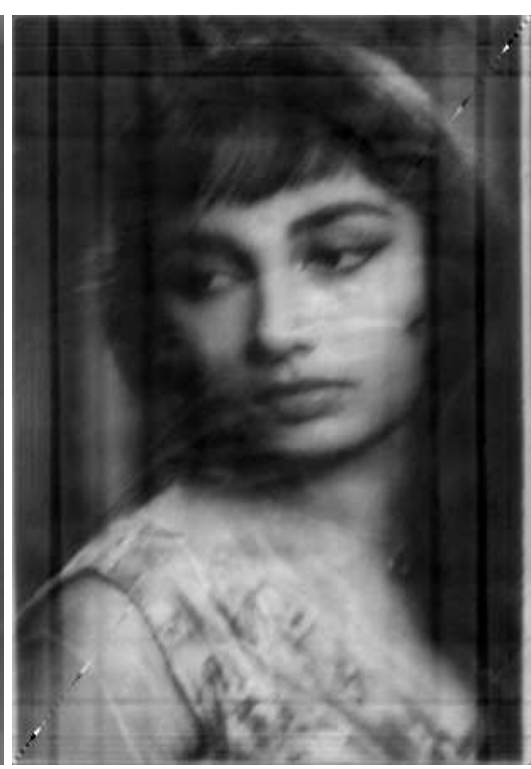

(b) Restored Image

Fig. 1 The restored result using our proposed method. 


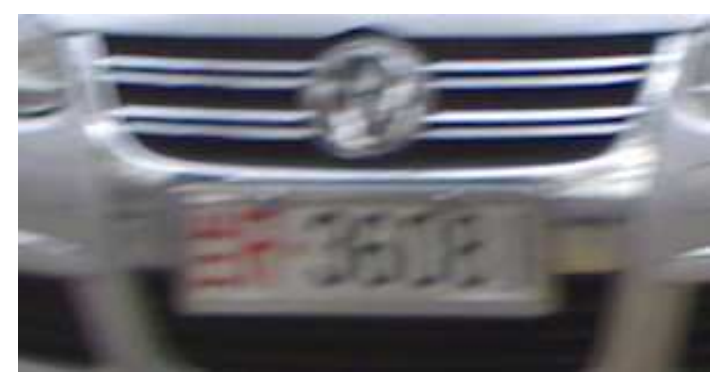

(a) Blurred Image

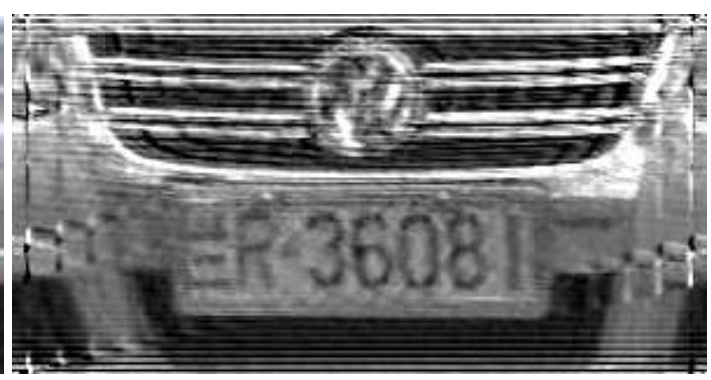

(b) Restored Image

Fig.2 The restored result using our proposed method.

\section{Conclusion}

This paper studies a motion blurred image processing method. The proposed method for motion blurred image has good recovery effect. The experiment results show that the E-d curve can be accurately estimate fuzzy length parameters; Based on the probability statistical method to estimate SNR parameters although in some errors, but use the image wiener filter recovery still can get very good result restores, wiener filtering recovery model of SNR parameters has strong robustness, through the smooth boundary the method can reduce the ringing effect, improve recovery effect.

\section{References}

[1] R. Gonzalez, R. Woods, Digital Image Processing (Second Edition), Publishing House of Electronics Industry, Beijing. 2007.

[2] F. Hiroko, Image restoration via wiener filtering in the frequency domain, WSEAS Transactions on Signal Processing. 5(2009) 63-73.

[3] W. Ming, X. Kong, L. Qu, Motion-blurred image restoration, Computer Engineering. 30(2004)133-135.

[4] W. Fawwaz, T. Shimahashi, M. Matsubara, S. Sugimoto, PSF estimation and image restoration for noiseless motion blurred images, Proceedings of the 7th WSEAS International Conference on Signal, Speech and Image Processing. (2007)1-7.

[5] W. Zeng, X. Lu, Region-based non-local means algorithm for noise removal, Electronic Letters. 47(2011)1125-1127.

[6] M. Zou, R. Unbehauen, An iterative method of blur identification and image restoration, Proceedings of the International Conference on Image Processing, Switzer land. (1996).

[7] W. Zeng, X. Lu, A generalized DAMRF image modeling for super-resolution of license plate, IEEE Transactions on Intelligent Transportation Systems 13(2012) 828-837. 